\title{
Quantum Encrypted Signals on Multiuser Optical Fiber Networks: Simulation Analysis of Next Generation Services and Technologies
}

\author{
Rameez Asif $\ddagger, *$ \\ ${ }^{\ddagger}$ Centre for Distributed Computing, Networks, and Security, School of Computing, \\ Edinburgh Napier University, Edinburgh (EH10 5DT), UK \\ *Corresponding Email: r.asif@napier.ac.uk
}

\begin{abstract}
Data encryption is gaining much attention these days from the research community and industry for transmitting secure information over access networks, i.e. 'fiber-to-the-home (FTTH)' networks and data centers. It is important that the newly designed encrypted networks are fully functional, reconfigurable, compatible, flexible and scalable with the existing deployed optical fiber networks around the globe. The prime benefit of having FTTH networks is the optical end-to-end data encryption that can best be implemented by quantum-keydistribution (QKD) protocols using state-of-the-art telecommunication components, i.e. continuous-variable quantum key distribution (CV-QKD). In this paper, we numerically investigate the quadrature phase shift keying (QPSK) based CV-QKD network that is compatible with the next generation (NG) services such as point-to-point (P2P) transmission and multicast overlay (MCO) traffic for audio/video signalling. We have further investigated the performance of quantum signals on multi-user fibers by emulating 7-, 12- and 19-core multi-core fibers (MCF). 100 Mbits/s secure key rates (SKRs) can be generated for inter-core crosstalk (XT) values of $\leq-20 \mathrm{~dB}$. These results and theoretical framework will allow the telecommunication industry to extend these scenarios not only to accommodate more complex trust constraints, but also to take into account the robustness and resiliency of a complex encrypted network.
\end{abstract}

\section{INTRODUCTION}

To satisfy the exponential demand of capacity requirements [1], new transmission technologies including digital signal processing have attracted many researchers over the last decade [2]. However, the fundamental limitations in single mode fiber (SMF) are imposed by the non-linear (NL) effects [3]. These impairments induce input power restrictions and reduced transmission distances. Therefore space division multiplexing (SDM), based on either multi-core fiber (MCF) or few-mode fiber (FMF), gives a promising solution to overcome these limitations [4]. High capacity MCF transmission has been investigated recently that scales the capacity and capacitydistance product (CDP) 10fold [5]-[7] till date. During last few years there have been concerted efforts invested in FMFs for long-haul transmission. The spatial modes generally exhibit differential mode group delay (DMGD) and differential modal loss/gain. To mitigate these linear impairments, equalization by multi-input multi-output (MIMO) digital signal processing (DSP) is required at receiver. On the contrary, there is an increasing interest in SDM based passive optical networks

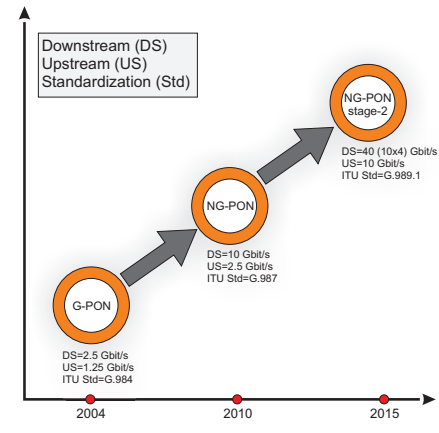

Fig. 1. Evolution of passive optical networks (PONs) w.r.t capacity and data rates per wavelength.

(PONs), towards larger splitting ratios (SRs) to increase coverage and reduce overall cost [8], [9] by incorporating lowmodal-crosstalk fiber so that discrete spatial modes can be detected individuality without using MIMO DSP [10]-[12] because it is cost-effective at access networking level not to use coherent receivers. In most practical systems, the limitation of having higher SRs is the power budget due to the fact that characteristically SMF splitters have an unavoidable bidirectional loss [13]. In order to avoid these losses, multiple feeder fibers are needed. This up-gradation will cause congestion in the installation ducts and laborious maintenance [14]. As an alternative, FMF-PONs have been demonstrated [11], [12] with 2-LP modes $\left(\mathrm{LP}_{01}, \mathrm{LP}_{11}\right)$ and bit-rates of $1.25 \mathrm{Gbit} / \mathrm{s}$ and $10 \mathrm{Gbit} / \mathrm{s}$, respectively.

To enable cutting-edge standardization of future optical access systems, the FSAN (Full Service Access Network) Group and ITU-T SG15 are currently discussing and standardizing the specifications of a $40 \mathrm{Gbit} / \mathrm{s}$ capable PON, which employs wavelength-division multiplexing (WDM) technology, for the purpose of enabling cost-effective $40 \mathrm{Gbit} / \mathrm{s}$ capable transmission capacity and multiple service capability [15]. This network architecture is termed as next-generation passive optical network stage-2 (NG-PON2), as described in Fig. 1 , that is which is going to be standardized in the ITU-T recommendation G.989 series [15]. Recently, we have demonstrated the first NG-PON2 SDM based network architecture by transmitting 6-modes each carrying $40 \mathrm{Gbit} / \mathrm{s}$ data for symmetric downstream (DS) and upstream (US) traffic [16]. Furthermore, it is also demonstrated that a distributed light 
source bank can be used for a colorless optical network unit (ONU) operation that will make wavelength management in NG-PON2 network much more easier and energy efficient [17]. Meanwhile, till date it is unclear which SDM fiber provides the best performance for NG-PON2, and in the future network operators may deploy different SDM fibers throughout the network [18].

Another benefit of the PONs is the optical end-to-end connectivity of the transmitter and receiver nodes. It is very much limited these days because of the presence of last-mile copper wires till the receiver node or subscriber. However, there are some successful implementation of optical end-toend networks in USA (source; Google Fiber Project), Spain (source: Telephonica), UAE (source: Du-Ericsson project). So we can say that fiber-to-the cabinet (FTTC) exists in the advanced broadband communications these days but fiber-tothe-home (FTTH) connections rarely exists. The researchers believe that FTTH is the key to develop a sustainable future, as it is now widely acknowledged that it is the only futureproof technology, when it comes to bandwidth capacity, speed, reliability, security and scalability [19]. In near future, it is envisaged that FTTH connections will exist, and a key offering would be the possibility of optical encryption that can best be implemented using quantum key distribution (QKD) techniques. QKD allows two legitimate parties to share a secret key that is known only to the parties, since the information leaked to an eavesdropper can be bounded as tightly as required. Hence, providing the information-theoretic secure keys. The main practical task however is to securely share the keys, especially when number of distant nodes, routers and switches are involved.

In this paper, we explore the design challenges in a QKD network to learn their ability to maintain the quality of service (QoS) under uncertainty, e.g. hybrid traffic with diverse power levels, inter-core crosstalk (XT) in NG fibers and resource allocation etc. Moreover, in order to realize longterm evolution (LTE) functionalities, i.e. protocol transparency and flexible scalability, the QKD network architecture should be investigated for point-to-point (P2P) and multicast overlay (MCO) video/audio services, both for performance and compatibility. By means of simulation, we analyse a QKD network generating $100 \mathrm{Mbits} / \mathrm{s}$ secure key rates by emulating the commercially available off-the-shelf equipment. The performance of next generation services, such as $\mathrm{P} 2 \mathrm{P}$ and MCO data scenarios are evaluated. Furthermore, the standard NG-PON data rates for QPSK classical data signals are used as hybrid traffic to analyse the performance of a QKD network. We also discuss the transmission performance of QKD signals in multiuser fiber, i.e MCF, to quantify the impact of next generation fiber infrastructures. The paper is arranged as follows: In Section II, we will discuss the numerical model for QKD network in detail, Section III will explain the impact of intercore crosstalk (XT) in dense core structures and in Section IV, we will discuss the results in detail. Finally we conclude the article followed by 'Appendix A' and 'Appendix B' to enlist the mathematical model of CV-QKD signals and signal
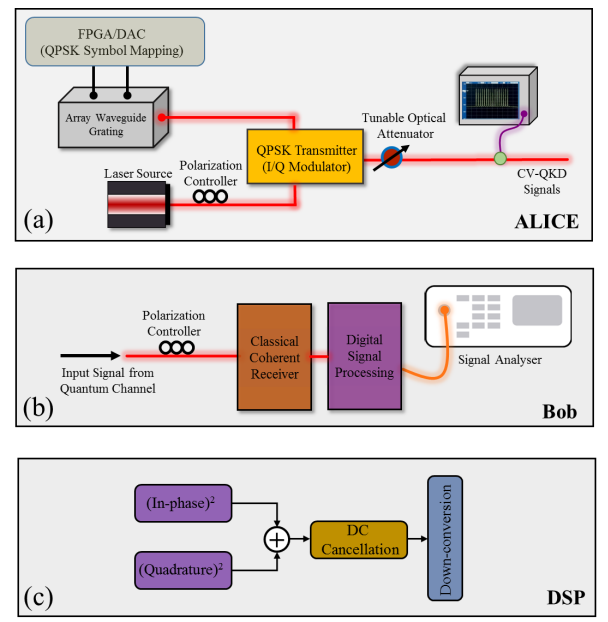

Fig. 2. Fundamental structure of the quantum network by employing commercial off-the-shelf telecommunication equipments: (a) transmitter (Alice), (b) receiver (Bob) and (c) digital signal processing (DSP) module to recover weakly powered quantum signals.

processing module (DSP), receptively.

\section{Simulation Model for Quantum Signals}

The simulation model of a simplified quantum-to-the-home (QTTH) network with quadrature phase shift keying (QPSK) based quantum transmitter (Alice) and local-local oscillator (LLO) based coherent receiver (Bob) is depicted in Fig. 2(a) and (b). At Alice, a narrow line-width laser is used at the wavelength of $1550 \mathrm{~nm}$ (standard telecommunication wavelength window) having a line-width of $\leq 5 \mathrm{kHz}$ allowing it to maintain low phase noise characteristics. A pseudorandom binary sequence (PRBS) of length $2^{31}-1$ is encoded for single channel transmission and delay de-correlated copies are generated for the WDM transmission. Furthermore, we perform pulse shaping at the transmitter according to the Nyquist criterion to generate Inter-symbol Interference (ISI) free signals. Resultant 1 GBaud QPSK (four-state phase-shift keying) signal is generated after the radio frequency (RF) signals are modulated via an electro-optical I/Q modulator, where $\mathrm{RF}$ frequency is kept at $2 \mathrm{GHz}$. The complete mathematical model of CV-QKD protocol is explained in 'Appendix A'. The modulation variance is modeled with the help of a variable optical attenuator (VOA) just before the quantum channel. We used the standard single mode fiber (SMF-28) parameters to emulate the quantum channel and losses. As the QKD transmission occurs at a very low power level, so the impact of optical Kerr effects are considered negligible. The polarization mode dispersion (PMD) is considered as $\leq 0.2 \mathrm{ps} \sqrt{\mathrm{km}}$ that enables more realistic simulations, i.e. comparative to the realworld deployed fiber networks.

The receiver module (Bob) consists of a $90^{\circ}$ optical hybrid, a high optical power handling balanced photo-diodes with $20 \mathrm{GHz}$ bandwidth. The responsivity, gain of TIA and noise equivalent power (NEP) of the receiver at $1550 \mathrm{~nm}$ is $0.8 \mathrm{~A} / \mathrm{W}$, $4 \mathrm{~K} . \mathrm{V} / \mathrm{W}$ and $22 \mathrm{pW} / \overline{\mathrm{Hz}}$, respectively. For our analysis, we have kept the high power, narrow line-width local oscillator 


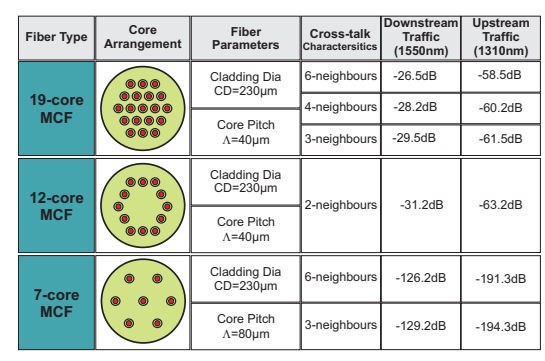

(a)

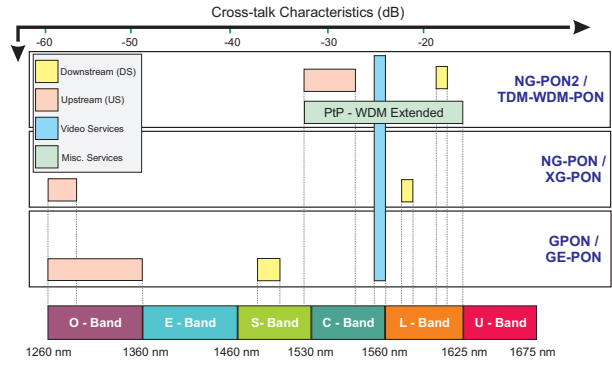

(b)

Fig. 3. (a) Performance comparison of diverse multi-core fibers w.r.t the inter-core crosstalk (XT) and (b) Wavelengths assignment for different next generation services for PON architectures.

TABLE I

Physical Parameters OF MCF to EVAluate XT AT $\lambda=1550$ NM AND $1310 \mathrm{NM}$

$\begin{array}{ll}\text { Parameter } & \text { Value } \\ \mathrm{n}_{o} & 1.44 \\ \mathrm{a}_{1} & 4.0 \mu \mathrm{m} \\ \Delta_{1} & 0.3 \% \\ \mathrm{a}_{2} & 8.0 \mu \mathrm{m} \\ \Delta_{2} & -0.7 \% \\ \mathrm{w}_{t r} & 4.0 \mu \mathrm{m} \\ \Lambda \text { (for 12- and 19-core MCF) } & 40 \mu \mathrm{m} \\ \Lambda \text { (for 7-core MCF) } & 80 \mu \mathrm{m}\end{array}$

at the receiver, i.e. integral part of Bob in-order to avoid any eavesdropping on the reference signal. That is why it is termed as local local oscillator (LLO). The LLO photon level is considered as $1 \times 10^{8}$ photon per pulse. A classical phase noise cancellation (PNC) based digital signal processing (DSP) is implemented to minimize the excess noise as shown in Fig. 2(c). The PNC stage has two square operators for in-phase and quadrature operators, one addition operator and a digital DC cancellation block assisted by a down-converter. The detailed implementation of the PNC module is explained in 'Appendix B' [24]. The coherent receiver requires a specific signal-tonoise-ratio (SNR) to detect the QPSK signal.

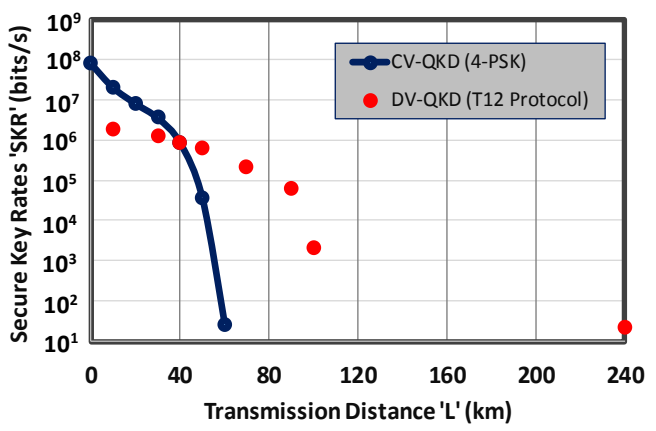

Fig. 4. Performance comparison of CV-QKD vs. discrete-variable (DV) QKD for access and metro networks with diverse transmittance values.

\section{WaVelength DePendent CRoss-Talk (XT) IN $\mathrm{MCF}$}

The main issue with next generation high capacity MCF transmission is, how we can increase the number of cores (thus, total capacity) while keeping the inter-core crosstalk (XT) low to allow error free and longer transmission distance since more dense cores (smaller core pitch $\Lambda$ ) normally increase the modes coupling among cores [25]. As a first step, we compared the XT behavior of 7-, 12- and 19- core MCF in the wavelength region of $1550 \mathrm{~nm}$ and $1310 \mathrm{~nm}$, as summarized in Fig. 3(a). These wavelengths are usually preferred for the DS and US operation of conventional PONs, as explained in Fig. 3(b). The analytical expression for measuring the XT of the fiber is explained in [26]. The refractive indices for the core, cladding and trench are $n_{1}, n_{0}$ and $n_{2}$, respectively, where the refractive indices in the $1^{\text {st }}$ and $2^{\text {nd }}$ claddings in trench-assisted structure are considered same. The core radius, the distance from the outer edge of the $1^{\text {st }}$ cladding to the core center, the distance from the outer edge of trench to the core center and trench width are $a_{1}, a_{2}, a_{3}$ and $w_{t r}$, respectively. The relative refractive index differences between core and cladding, trench and cladding are $\Delta_{1}$ and $\Delta_{2}$, respectively. For formulation of XT in this paper, over a transmission length of $20 \mathrm{~km}$, cladding diameter (CD) of $230 \mu \mathrm{m}$ and cladding thickness (CT) of $35 \mu \mathrm{m}$ following values are used as enlisted in Tab. I.
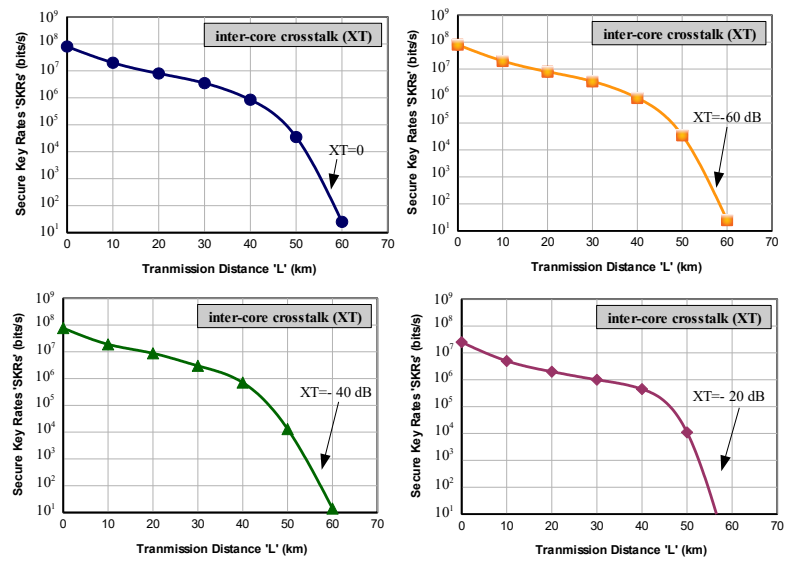

Fig. 5. Dependence of secure key rates on inter-core crosstalk (XT) .

\section{RESULTS AND DISCUSSIONS}

\section{A. Point-to-Point Transmission in Single Mode Fiber}

Based on the numerical model as described in Section II, we extended our studies to calculate the secure key rates (SKR) at 


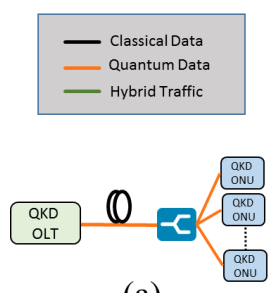

(a)

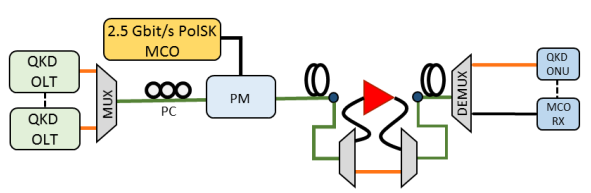

(c)

Fig. 6. Fundamental architecture of: (a) QKD access network, (b) Hybrid classical-quantum access network and (c) QKD access network with pointto-point $(\mathrm{P} 2 \mathrm{P})$ and multicast overlay $(\mathrm{MCO})$ video/audio services.
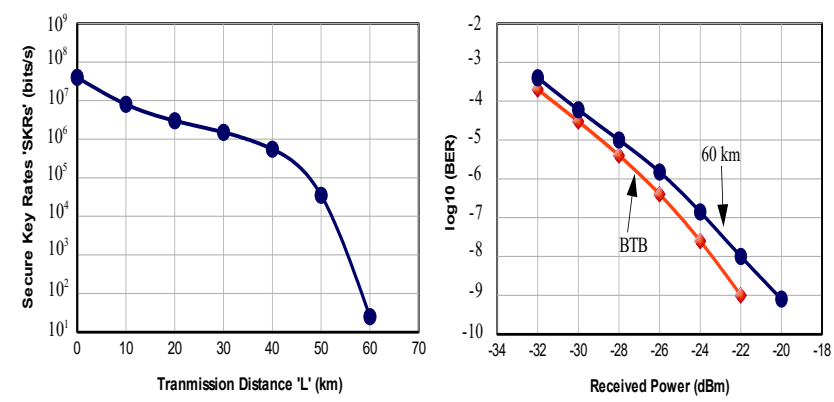

Fig. 7. Simulated system performance of QKD network with encrypted P2P and classical MCO signaling.

different transmission distances, i.e. transmittance values. Simulations are performed by assuming $60 \%$ detector efficiency and $95 \%$ reconciliation efficiency. The results are depicted in Fig. 4. The maximum of $100 \mathrm{Mbits} / \mathrm{s}$ SKR can be achieved with this configuration by employing commercial off-the-shelf modules for transmittance $(\mathrm{T})=1$ for QPSK modulation. While SKR of $\approx 25 \mathrm{Mbits} / \mathrm{s}$ and $1 \mathrm{Mbits} / \mathrm{s}$ at $\mathrm{T}=0.8$ and 0.6 , respectively. A comparison of distance dependent secure key generation rate between CV-QKD using $20 \mathrm{GHz}$ balanced homodyne detector and state-of-the-art DV-QKD systems based on T12 protocol [27] is shown in Fig. 4. The transmission distance of CV-QKD systems, limited by the lack of advanced reconciliation techniques at lower SNR, is far lower than for DV-QKD demonstrations. However, comparison of DV-QKD and $\mathrm{CV}-\mathrm{QKD}$ shows that CV-QKD has the potential to offer higher speed secure key transmission within an access network area $(100 \mathrm{~m}$ to $50 \mathrm{~km})$. Especially from $0-20 \mathrm{~km}$ range, i.e. typical FTTH network, the SKR generated by using the traditional telecommunication components are 10s of magnitude higher than that of DV systems. From the results, we can depict that CV-QKD protocol is ideal for access networks, while it is fully compatible with existing telecommunication infrastructure, cost effective, energy efficient and scalable.

\section{B. Point-to-Point Transmission in Multicore Fiber}

As we have discussed earlier the impact of inter-core crosstalk in next generation multi-user fibers, i.e. MCF, in Section III. It is evident that the dense core structures will impact the performance of the transmission system, especially for the cores located around the central axis of the cladding. Therefore, we have extended our CV-QKD studies and quantified the impact of crosstalk on secure key rates. The results are depicted in Fig. 5. It can be seen that the crosstalk factor from 7-core and 12-core fiber has no impact on the performance of QKD system. While there is negligible performance penalty with the crosstalk value of $-20 \mathrm{~dB}$. Therefore, next generation multi-user fibers can easily be used for quantum traffic due to their comparative performance as compared to standard single mode fibers. This will give the network two main advantages: (a) aggregate secure key rates will be increased exponentially with the increasing number of cores and (b) power budget of the network will be improved due to the fact that next generation fibers are using wave-guide based splitters and combiners that inherently have less insertion loss (typically in the range of $\leq 1 \mathrm{~dB}$ ) as compared to standard passive splitters.

\section{QKD Network with Next Generation Services}

In order to deploy the QKD network with existing access/broadband infrastructure, the quantum signals should show full compatibility with the next generation network standards and services, as shown in Fig. 6. To analyze the transmission of point-to-point (P2P) encrypted transmission and classical multicast overlay (MCO) signals we modified the numerical model as described in Section II. The optical line terminal (OLT), four WDM QPSK encrypted QKD signals are generated for downstream transmission and multiplexed signals are sent for the MCO modulation with classical data traffic. These signals are fed into a PolSK modulator that consists of a polarization controller (PC) and a phase modulator (PM). By optimizing PC to fit the input state of polarization (SOP), PolSK multicast overlay data can be added onto the combined signals. The data rate of PolSK classical signals are kept at $2.5 \mathrm{Gbits} / \mathrm{s}$. The P2P signals are then detected by homodyme coherent receiver with local local oscillator (LLO) while MCO signals are fed to PolSK receiver having a polarization beam splitter (PBS) that performs the conversion from polarization modulation to intensity modulation. An electrical low pass filter (EF) is also used which can eliminate the crosstalk between the P2P signals and MCO signals. We would also like to mention that the optical amplifiers are only available on the classical data paths to regenerate the signals, if needed for long range transmission. The results are depicted in Fig. 7. The SKRs of the quantum signal shows negligible degradation, while the non-zero key threshold is at $60 \mathrm{~km}$. On the other hand the $2.5 \mathrm{Gbits} / \mathrm{s}$ PolSK MCO signals are successfully detected at $10^{-9}$ BER value after the transmission distance of $60 \mathrm{~km}$. 


\section{CONCLUSION}

To summarize, we have investigated the high secure key rate based CV-QKD network in detail. The results depict that $\mathrm{CV}-\mathrm{QKD}$ protocol is fully compatible with the multi-user next generation fibers and $100 \mathrm{Mbits} / \mathrm{s}$ secure key rates (SKRs) can be generated for inter-core crosstalk (XT) values of $\leq-20 \mathrm{~dB}$. This up-gradation can provide two-fold advantage: (a) upto 1 Gbits/s aggregate SKRs can be achieved by using multiple multiplexing techniques by exploiting the physical properties of wavelength, space and time, i.e. multi-dimensional QKD protocol and (b) power budget of the network will be improved due to the fact that next generation fibers are using waveguide based splitters and combiners that inherently have less insertion loss (typically in the range of $\leq 1 \mathrm{~dB}$ ) as compared to standard passive splitters. Furthermore, next generation services such as $\mathrm{P} 2 \mathrm{P}$ and $\mathrm{MCO}$ are investigated and we have observed negligible degradation in the SKRs. These results will allow the telecommunication companies to integrate the QKD services, especially for banks, e-health applications, research institutes, data centers and military communications, in their existing broadband infrastructures and these solutions are viable, robust, cost-effective and energy efficient.

\section{ACKNOWLEDGEMENT}

The authors would like to acknowledge the project (grant no. 830965) research funding from Edinburgh Napier University, UK for project STRENGTH (Scalable, Tuneable, Resilient and Encrypted Next Generation Transmission Hub). We would also like to say thanks to Prof. Alan Woodward from University of Surrey, UK for his valuable discussions on future QKD networks.

\section{APPENDIX A}

\section{Mathematical Model for CV-QKD Signals}

Alice generates random m-PSK symbols that can be optimized from pseudo-random binary sequence (PRBS) at the transmitter, i.e $\mathrm{I}(\mathrm{t}), \mathrm{Q}(\mathrm{t}) \in\{-1,+1\}$. These random symbols are up-converted to radio-frequency (RF) domain with corresponding in-phase and quadrature signals [20], that are denoted by $S_{I}(t)$ and $S_{Q}(t)$. Mathematically these two components can be expressed as in Eq. 1 and 2.

$$
\begin{gathered}
S_{I}(t)=I(t) \cos \left(\omega_{1} t\right)-Q(t) \sin \left(\omega_{1} t\right) \\
S_{Q}(t)=I(t) \sin \left(\omega_{1} t\right)+Q(t) \cos \left(\omega_{1} t\right)
\end{gathered}
$$

where, $\omega_{1}$ is the RF angular frequency. The output is then used as the input of I/Q modulator, Mach-Zehnder modulator (MZM). The resultant optical field can be expressed as in Eq. 3 and further be simplified as in Eq. 4.

$$
\begin{array}{r}
E(t)=\left\{\cos \left[A S_{I}(t)+\frac{\pi}{2}\right]+j \cos \left[A S_{Q}(t)+\frac{\pi}{2}\right]\right\} \\
\sqrt{P_{s}} e^{j\left[\omega t+\varphi_{1}(t)\right]}
\end{array}
$$

$$
\begin{array}{r}
E(t) \simeq \sqrt{2 P_{s}} e^{j\left[\omega t+\frac{j \pi}{4}\right]}-A \cdot[I(t)+j Q(t)] \\
\sqrt{2 P_{s}} e^{j\left[\left(\omega+\omega_{1}\right) t+\varphi_{1}(t)\right]}
\end{array}
$$

where, $A$ refers to the modulation index; $\mathrm{P}_{s}, \omega$ and $\varphi_{1}(t)$ represent the power, angular frequency of the carrier and phase noise. For evaluating the modulation variance $V_{A}$ of the optical signal, expressed as shot-noise-units (SNUs), the parameter $A$ and variable optical attenuator (VOA) are modelled. To further simply the mathematical model, the quantum channel loss is expressed as the attenuation of the optical fiber. Moreover, channel introduced noise variance is expressed as in Eq. 5.

$$
\chi_{\text {line }}=\frac{1}{T}+\epsilon-1
$$

where, $T$ is the transmittance (relation between transmission length and attenuation i.e. $\mathrm{T}=1$ for back-to-back and $\mathrm{T}=0.2$ for $80 \mathrm{~km}$ fiber transmission) and $\epsilon$ is the excess noise. Practically, possible excess noise contributions, expressed as SNUs [21], [22], may come from the imperfect modulation, laser phase noise, laser line width, local oscillator fluctuations and coherent detector imbalance [23].

In this paper, we have used the concept of a local local oscillator (LLO). It is a very vital configuration to keep the laser at the receiver, i.e. Bob's side, in-order to prevent any eavesdropping attempt on the quantum channel to get the reference information of the incoming signal. The electric field of the LLO can be expressed as in Eq. 6.

$$
E_{L L O}(t)=\sqrt{P_{L L O}} e^{j\left[\omega_{L L O} t+\varphi_{2}(t)\right]}
$$

where, $\mathrm{P}_{L L O}, \omega_{L L O}$ and $\varphi_{2}(\mathrm{t})$ represents the power, angular frequency and phase noise of the LLO, respectively. The structure of the Bob comprises of a $90^{\circ}$ optical hybrid and two balanced photo-detectors. The coherent receiver has an overall efficiency of $\eta$ and electrical noise of $\mathrm{V}_{e l}$. Practically, $\mathrm{V}_{e l}$ comprises of electrical noise from trans-impedance amplifiers (TIA) as well as contribution from the analogue-to-digital converters (ADCs). The receiver added noise variance can be expressed as in Eq. 7.

$$
\chi_{\text {det }}=\frac{\left(2+2 V_{e l}-\eta\right)}{\eta}
$$

Furthermore, the total noise variance of the system, including Alice and Bob, can be expressed as in Eq. 8.

$$
\chi_{\text {system }}=\frac{\chi_{\text {line }}+\chi_{\text {det }}}{T}
$$

\section{APPENDIX B}

\section{Digital Signal Processing (DSP) Module}

Conventionally, in-order to detect the weakly powered incoming quantum signals, a high power local oscillator is required. It is very important to select the local oscillator with narrow line width, so that the laser fluctuations cannot contribute to the system excess noise. Furthermore, it will help the coherent receiver to have a low complex digital 
signal processing (DSP) module, i.e. phase noise cancellation (PNC) algorithm. As a prerequisite for PNC module, the photo-currents of the in-phase and quadrature signals, after the balanced photo-detectors, have to be measured accurately. Mathematically, they can be expressed as in Eq. 9 and 10.

$$
\begin{array}{r}
i_{I}(t) \propto \sqrt{2} \cos \left[\left(\omega-\omega_{L O}\right) t+\varphi_{1}(t)-\varphi_{2}(t)+\frac{\pi}{4}\right] \\
-A I(t) \cos \left[\left(\omega+\omega_{1}-\omega_{L O}\right) t+\varphi_{1}(t)-\varphi_{2}(t)\right] \\
+A I(Q) \cos \left[\left(\omega+\omega_{1}-\omega_{L O}\right) t+\varphi_{1}(t)-\varphi_{2}(t)\right]+n_{I} \\
i_{Q}(t) \propto \sqrt{2} \sin \left[\left(\omega-\omega_{L O}\right) t+\varphi_{1}(t)-\varphi_{2}(t)+\frac{\pi}{4}\right] \\
-A I(t) \cos \left[\left(\omega+\omega_{1}-\omega_{L O}\right) t+\varphi_{1}(t)-\varphi_{2}(t)\right] \\
+A I(Q) \cos \left[\left(\omega+\omega_{1}-\omega_{L O}\right) t+\varphi_{1}(t)-\varphi_{2}(t)\right]+n_{Q}
\end{array}
$$

where, $\mathrm{n}_{I}$ and $\mathrm{n}_{Q}$ defines the in-phase and quadrature components of the additive phase noise, that needs to be compensated. We have implemented the phase noise cancellation (PNC) algorithm [20]. By combining the squares of the inphase and quadrature component of photo-currents, as in Eq. 9 and 10 , i.e. $\mathrm{i}_{I}^{2}(\mathrm{t})+\mathrm{i}_{Q}^{2}(\mathrm{t})$, and canceling the DC component the final result can be expressed as in Eq. 11.

$$
\begin{array}{r}
i_{S}(t) \propto 2 \sqrt{2} A I(t) \cos \left(\omega_{1} t-\frac{\pi}{4}\right)+2 \sqrt{2} A Q(t) \cos \left(\omega_{1} t-\frac{\pi}{4}\right) \\
+2 \sqrt{2}\left\{n_{I} \cos \left[\left(\omega-\omega_{L O}\right) t+\varphi_{1}(t)-\varphi_{2}(t)+\frac{\pi}{4}\right]\right. \\
\left.n_{Q} \sin \left[\left(\omega-\omega_{L O}\right) t+\varphi_{1}(t)-\varphi_{2}(t)+\frac{\pi}{4}\right]\right\}
\end{array}
$$

The final step in the DSP module is to down-convert the RF signal. The resultant in-phase and quadrature components can be expressed as in Eq. 12 and 13

$$
\begin{gathered}
r_{I}=\operatorname{LPF}\left[i_{S}(t) \cos \left(\omega_{1} t-\frac{\pi}{4}\right)\right]=-\sqrt{2} A I+n_{I}^{\prime} \\
r_{Q}=L P F\left[i_{S}(t) \sin \left(\omega_{1} t-\frac{\pi}{4}\right)\right]=-\sqrt{2} A Q+n_{Q}^{\prime}
\end{gathered}
$$

where, n' ${ }_{I}$ and n' ${ }_{Q}$ are the equivalent additive noise that is added during the transmission and detection processes prior to DSP module. By considering Eq. 12 and 13, it is concluded that the original m-PSK signals can be detected without any frequency and phase distortions.

\section{REFERENCES}

[1] P.P. Mitra and J.B. Stark, "Nonlinear limits of the information capacity of optical fiber communication", Nature, 411, 1027-1030 (2001).

[2] J.R Essiambre, G.J. Foschini, G. Karmer and P.J. Winzer, "Capacity limits of information transport in fiber-optic networks", Physics Review Letters, 101, 163901 (2008).

[3] C. Lin, R. Asif, M. Holtmannspoetter, and B. Schmauss, "Nonlinear mitigation using carrier phase estimation and digital backward propagation in coherent QAM transmission," Optics Express, 20, B405-B412 (2012).

[4] T. Morioka, "New generation optical infrastructure technologies: EXAT initiative towards 2020 and beyond", Proc. OECC, FT4 (2009).
[5] T. Mizuno et al., "12-core $\times 3$-mode dense space division multiplexed transmission over $40 \mathrm{~km}$ employing multi-carrier signals with parallel MIMO equalization", Proc. OFC, PDP Th5B.2 (2014).

[6] H. Takara et al., "1.01-Pb/s (12 SDM/222 WDM/456 Gb/s) cross-talk managed transmission with $91.4-\mathrm{b} / \mathrm{s} / \mathrm{Hz}$ aggregate spectral efficiency," in Proc. ECOC, Th.3.C.1 (2012).

[7] T. Kobayashi et al., " $2 \times 344 \mathrm{~Tb} / \mathrm{s}$ propagation-direction interleaved transmission over $1500 \mathrm{~km}$ MCF enhanced multi-carrier full electric field digital back-propagation" in Proc. ECOC, PDP 3.E.4 (2013).

[8] B. Zhu et al., "Seven-core multicore fiber transmissions for passive optical network," Opt. Express, vol. 18, no. 11, p. 11117-11122 (2010).

[9] B. Li et al., "Experimental demonstration of large capacity WSDM optical access network with multi-core fibers and advanced modulation formats," Opt. Express, vol. 23, no. 9, p. 10997-11006 (2015).

[10] C. Xia et al., "Demonstration of World's First Few-Mode GPON", Proc. ECOC, PD.1.5, Cannes (2014).

[11] T. $\mathrm{Hu}$ et al., "Experimental Demonstration of Mode-DivisionMultiplexing and Time-Division-Multiplexing Passive Optical Network," Proc. ACP, AF4B.7, Shanghai (2014).

[12] T. Hu et al., "Experimental Demonstration of Passive Optical Network Based on Mode-Division-Multiplexing," Proc. OFC, Th2A.63, Los Angeles (2015).

[13] C. Xia et al., "TDMA Few-Mode Passive Optical Network," Proc. ACP, paper ATh2F.2, Shanghai (2014).

[14] R. Asif et al., "Flexible Space Division Multiplexed WDM-PON with 16-QAM Point-to-Point and PolSK Modulated Multicast Overlay Services," Proc. CLEO Europe, Cl.P.10, Munich (2015).

[15] Kota Asaka and Jun-ichi Kani, "Standardization Trends for NextGeneration Passive Optical Network Stage-2 (NG-PON2)," NTT Technical Review, vol. 13, no. 3, March (2015).

[16] R. Asif et al., "Experimental Demonstration of 6-Mode Division Multiplexed NG-PON2: Cost Effective $40 \mathrm{Gbit} / \mathrm{s} /$ Spatial-Mode Access Based on 3D Laser Inscribed Photonic Lanterns," Proc. ECOC, Tu.1.5.1 (2015).

[17] Z. Feng et al., "Multicore-Fiber-Enabled WSDM Optical Access Network With Centralized Carrier Delivery and RSOA-Based Adaptive Modulation,” IEEE Photonics Journal, vol.7, no.4, pp.1-9, Aug (2015).

[18] N. Fontainee et al., "Heterogeneous Space-Division Multiplexing and Joint Wavelength Switching Demonstration," Proc. OFC, Th5C.5, Los Angeles USA (2015)

[19] C. F. Lam,"Fiber to the home: Getting beyond 10 gigabit/sec," Opt. Photon. News, vol. 27, no. 3, pp. 2229, Mar (2016).

[20] Z. Qu, I. B. Djordjevic, and M. A. Neifeld, "RF-subcarrier-assisted fourstate continuous-variable QKD based on coherent detection," Opt. Lett., vol. 41, no. 23, pp. 55075510, Dec (2016).

[21] B. Qi, L.-L. Huang, L. Qian, and H.-K. Lo, "Experimental study on the Gaussian-modulated coherent-state quantum key distribution over standard telecommunication fibers," Phys. Rev. A, vol. 76, p.052323, Nov (2007).

[22] S. Fossier, E. Diamanti, T. Debuisschert, A. Villing, R. TualleBrouri, and P. Grangier, "Field test of a continuous-variable quantum key distribution prototype," New Journal of Physics, vol. 11, no. 4, p. 045023, (2009).

[23] D. Huang, D. Lin, C. Wang, W. Liu, S. Fang, J. Peng, P. Huang, and G. Zeng, "Continuous-variable quantum key distribution with 1 megabit per second secure key rate," Opt. Express, vol. 23, no. 13, pp. 1751117 519, Jun (2015).

[24] R. Asif and W.J. Buchanan, "Seamless cryptographic key generation via off-the-shelf telecommunication components for end-to-end data encryption," 10th IEEE International Conference on Internet of Things (iThings), paper ID: SITN2, Exeter, UK, Jun (2017).

[25] R. Asif et al., "Experimental Demonstration of 6-Mode Division Multiplexed NG-PON2: Cost Effective $40 \mathrm{Gbit} / \mathrm{s} / \mathrm{Spatial}-M o d e$ Access Based on 3D Laser Inscribed Photonic Lanterns," Proc. ECOC, Tu.1.5.1, Valencia, Spain (2015).

[26] R. Asif, "Advanced and flexible multi-carrier receiver architecture for high-count multi-core fiber based space division multiplexed applications," Scientific Reports, vol. 6, p. 27465, Jun (2016).

[27] B. Froehlich, M. Lucamarini, J. F. Dynes, L. C. Comandar, W. W.- S. Tam, A. Plews, A. W. Sharpe, Z. Yuan, and A. J. Shields, "Long-distance quantum key distribution secure against coherent attacks," Optica, vol 4, no. 1, pp. 163167, Jan (2017). 